\title{
Adapting to Climate Variability Impacts on Coastal Zone for Small Islands - Case Study on the Azores Archipelago
}

\author{
Kiat $\mathrm{Ng}$,
}

Climate variability is becoming a significant concern for Small Islands (SI). Based on historical records, experience with current patterns and consequences of climate variability and climate model projections, SI have legitimate concerns about their future due to their inherently high vulnerability and low adaptive capacity (IPCC, 2007). With limited land availability and exposure to ocean from all sides, coastal zones which are highly vulnerable to Climate Variability Impacts (CVI) are of vital importance to SI. Hence, there is an urgent need for SI to be prepared for future CVI on their coasts.

The Azores, a Portuguese Autonomous Region, is a group of nine volcanic islands located in North Atlantic. Coastal Zone Management Plans (CZMPs) have been developed for four of the islands to preserve coastal resources and protect well-being of the residents. Studies postulated a slight temperature increase in the Azores due to projected increase in greenhouse emissions, with the postulated biggest impact being increase of precipitation in winter and decreases in other seasons (Calado et al., 2009). With climate variability and global climate change policies potentially exerting significant effects on the Azores, the Azores needs to strengthen its resilience through reducing its vulnerability to CVI such as sea-level change, landward seawater intrusion, and increases in coastal erosion $(0.8 \mathrm{~m} / \mathrm{yr}$ in some places), coastal storms, stream flooding and landslides (Calado et al., 2009). These potential coastal hazards as a result of CVI need to be better understood in order to establish appropriate adaptation strategies and mitigation measures.

It is important for the Azores to be prepared for future climate variability because it will stretch Azores's inherent vulnerable coasts beyond its resilience limit. Thus, more studies need to be carried out on CVI on the coasts, in order to strengthen its CZMPs for the future through integrating adaptation strategies and mitigation measures. 